\title{
We André Roumieux (1932-2020)
}

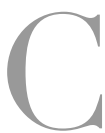

e Dimanche 19 avril 2020 André s'en est allé. C'est avec un sourire malicieux qu'il nous dirait avoir “ rendu son chapeau claque ” pour retourner à la vie civile et se rendre dans son Lot natal, précisément à Mayrinhaa-Lentour. Un retour au pays pour y couler une retraite paisible autant qu'active, un moment quand même pas trop attendu sinon craint. Infirmier de circonstance, avant de l'être de profession, et après plus de trois décennies dans cet hôpital qui avait su l'accueillir, et dont il avait su écrire l'histoire associée à celle de sa discipline : la psychiatrie dont il fut un acteur impliqué, André fut un infirmier autodidacte a t-on dit? un infirmier écrivain assurément qui voyait en chacun de nous un producteur de réflexions et d'écrits, tout comme lui, s'adressant à l'un ou l'autre d'entre nous avec cette question : “ qu'est-ce que tu as sur le feu en ce moment? " Ceci pour nous dire sa conviction que tous, nous étions avec lui sur ces terrains de la recherche et des projets en cours. Dans son premier ouvrage : “je travaille a l'Asile d'aliénés » paru en 1974 il nous dit son choix du travail en Psychiatrie, posé comme " concours de circonstances " un concours qui le conduisit à aiguiser sa curiosité et à dire quelque chose sur les mœurs de l'Asile et ce dans un climat anti psychiatrique finissant : le temps du désaliénisme, le temps des Sivadon ou celui de Madame Chaigneau pour ne citer que ces deux figures de la psychiatrie. Un premier ouvrage qui le consacra comme professionnel et surtout écrivain en cette fin d'après midi dans les locaux du " temple de la psychiatrie » : l'hopital Sainte Anne. Un second ouvrage devait suivre, au titre évocateur : “ La tisane et la camisole » avant de s'orienter résolument dans une voie de chercheur et de clinicien en quête de vérité, celle de Antonin Artaud auquel il sut consacrer deux ouvrages dont le dernier : “ Artaud et L'Asile »publié en 2015 restitue les échanges et la souffrance d'Antonin au prises avec son histoire, celle du couple parental, celle des liens avec mère et grand mère, celle aussi de ses relations avec le corps médical du temps de Rodez et de ses liens avec le docteur G Ferdière qui sut l'accompagner dans bien des circonstances. Ce qu'on voudrait retenir de ce fondateur de la SERHEP : le musée de Ville-Évrard, c'est que cet homme de grande culture qui n'avait d'égal que sa modestie, ne manquait quand même pas d'humour sur ses ses origines rurales, notamment quand il quittait ses lunettes, nous déclarant avec le plus grand sérieux : “ on peut enlever un p’tit gars 
de la campagne, mais pas la campagne d'un p'tit gars ». Un homme fidèle et auquel nous le resteront... fidèle.

S.G. Raymond

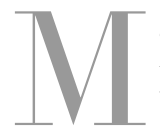

a rencontre avec André Roumieux est ancienne et pour moi infiniment précieuse. Elle eut lieu au pavillon des “ Tilleuls » de l'EPS de Ville-Évrard, secteur 10 que dirigeait à l'époque d'une main de fer Mm le Dr. Goujon. André était surveillant de ce pavillon dont il fit rapidement son fief. Nous étions au cour des années 1980, je venais de soutenir ma thèse, petit objet bancal traitant des adolescents de famille d'origine maghrébine en France, qui se voulait une recherche interculturelle précise et froide et fut un peu mieux que cela, trouvant sa bifurcation féconde par la relation d'une prise en charge d'un jeune adulte qu'on dira d'origine algérienne admis à Ville-Évrard. Je ne pus écrire cette relation clinique que parce que mon travail était soutenu dans ce pavillon. André Roumieux avait réuni autour de lui une solide équipe d'infirmiers, tous ou presque venus sur Sud-Ouest, et tous au départ un peu ironiques devant le jeune psychologue que j'étais tout frais tartiné des diplômes requis et prolixe en de tics de langage impayables qu'on attrape à l'université et dont la pratique nous débarbouille rapidement. Puis ils devinrent attentifs, accueillants sans perdre rien de leur humour salubre. Mon alliance avec André et son équipe se scella ce midi, dans le bureau des infïmiers où nous nous partagions jambons, saucissons et fromages venus du Périgord Noir et du Lot. Quoi de mieux qu'un bon repas totémique pour se sentir conquis par l'esprit de corps. Alors je partageais mes emballements, mes hypothèses, et m'entretenais avec les uns et les autres des aléas des prises en charge, comme on le dit, et c'est bien avec l'écoute d'André Roumieux et de ses vaillants commensaux que je réalisa, peu à peu, l'importance de l'institution, de l'histoire, la place de la subjectivité du soignant dans le soin, le sens du collectif. Sans cette écoute, je n'aurais pas su mener certains suivis dont celui de ce jeune patient halluciné et déraciné qui humanisa ma thèse. Il faut dire aussi qu'à mon enthousiasme désordonné et encombrant, les excellents psychiatres qui tenaient la maison réservaient une attention limitée et un accueil tout de douche froide.

La vie institutionnelle c'est aussi des drames, des patients qui meurent, des violences. Point tant que cela, mais tout de même. Et toujours la vivacité d'André Roumieux, son humanité, qui me permettait de parler de mes doutes, de mes angoisses sans m’en sentir trop encombré ou trop inhibé.

André Roumieux portait avec sagesse et bonhomie la vertu de l'accueil et l'audace de la parole. Grâce à lui, les infirmiers et même le psychologue turbulent avaient leur mot à dire. Gardienne de l'histoire, son équipe savait se faire pionnière avec une efficacité dont nul ne se fit gloire. La joie du travail bien fait, le goût 
d'expérimenter ces trouvailles vivantes du secteur (Visite à domicile, etc.) réchauffait nos échanges.

Sur le métier d'infirmier peu ont voulu et su écrire comme André Roumieux. Je tiens pour indispensable pour qui aujourd'hui bataille pour une psychiatrie humaniste la lecture de ses deux livres majeurs, Je travaille à l'asile d'aliénés (1974) et La tisane et la camisole (1981). Bibliophile, amoureux du surréalisme, grand connaisseur d'Artaud avec qui il avait une relation presque fraternelle, toute sa vie il s'est battu contre la contention, les soins trop lourds ou trop hâtifs.

Sans André Roumieux, aurais-je continuer à travailler en psychiatrie? Aujourd'hui, je sais bien que non. Ma dette est importante.

Olivier Douville 